\title{
Magnetic Strips as Landmarks for Mobile Robot Navigation
}

\author{
Pavel Škrabánek \\ Faculty of Electrical Engineering and Informatics \\ University of Pardubice \\ Studentská 95, 53210 Pardubice \\ Email: pavel.skrabanek@upce.cz
}

\author{
Pavel Vodička \\ Faculty of Electrical Engineering and Informatics \\ University of Pardubice \\ Studentská 95, 53210 Pardubice \\ Email: st39674@student.upce.cz
}

\begin{abstract}
In this paper, a low-cost localization system designed for mobile robots is introduced. The system has been developed for a teaching aid where a mobile robot operates in a maze. The mobile robot is navigated by a high level control system where path-planning is executed; however, autonomous execution of the plan is required from the robot. The robot uses magnetic landmarks, among others, for its localization. Detection of the landmarks is realized by a triple axis magnetometer sensor. The landmarks are made from a commercially available magnetic sheet. Construction of the sheets limits this approach. A successful application has to meet some requirements which are described in this paper.

Keywords - mobile robots; educational robots; robot sensing systems; magnetic sensors
\end{abstract}

\section{INTRODUCTION}

Mobile robots are popular within the education system. Many different types of mobile robots suitable for education have been introduced. They are employed in different environments where diverse activities are required to be performed, e.g. line following [1], collecting stuff, obstacle avoidance, or solving a maze [2].

Development of a teaching aid aimed at use of a mobile robot is a complex process. The process can be considerably simplified once a commercial solution is used, e.g. popular kit LEGO Mindstorms [3], or a product of a Swiss company K-Team Corporation [4], [5]. Although many different commercial products are available on the market, it is difficult to find an inexpensive solution which perfectly fits a specific teaching purpose. Of course, it is possible to use a virtual environment [6]; however, students lose the connection with the reality.

The good experience with mobile robots while teaching path-planning techniques [2] motivated us to enrich our courses on artificial intelligence with a practical exercise based on this experience. The lack of inexpensive solutions suitable for this purpose led us to develop a new teaching aid tailored to our requirements.

This teaching aid consists of a maze, a camera system and a mobile robot. Since a semi-autonomous behavior of the robot is required, robot localization had to be solved during aid development. Since the robot operates in a structured indoor environment, a ISBN 978-80-261-0602-9 (c) University of West Bohemia, 2016 localization method based on artificial landmarks can be used beneficially.

Localization is an important issue in mobile robotics; thus, the number of published localization methods is not surprising. Vision based systems [7], systems using invisible barcodes [8], or radiofrequency identification tags [9] can be given as examples. Considering the required low-price of the teaching aid, an inexpensive localization system has to be used in our case; however, the use of the camera system brings some limitations. In our opinion, a localization system based on magnetic landmarks meets both the requirements and the restrictions.

Localization systems based on magnetic landmarks have been proven in many applications [10], [11], [12]. In our approach, landmarks made up of commercially available magnetic sheets are used for indication of cell borders. Accordingly, the mobile robot is equipped by a low-price triple axis magnetometer sensor. Although implementation of such localization system seems to be simple, our practical experience is different. In this paper, you can becomed familiarized with the localization system as well as with our insights obtained during its implementation.

The rest of the paper is organized in this way. The teaching aid and purpose of its usage is introduced in section II. Concept of the localization system is formulized in the same section. Implementation of the system and its verification is described in section III. The work is summarized in section IV.

\section{CONCEPT OF THE TEACHING AID AND LOCALIZATION}

The teaching aid is designed as a supporting tool while teaching path-planning techniques in a course of artificial intelligence, which is lectured to graduate students. It consists of the maze, the camera system, the mobile robot and a high level control system. Since the first three parts are important for understanding of the teaching aid concept, they are described in necessary details in subsections II-A, II-B, and II-C. The question of the path-planning and implementation of the plan is opened in subsection II-D. Finally, concept of the localization system is introduced in subsection II-E. 


\section{A. Maze}

In terms of path-planning, the maze is a working space of the mobile robot. The maze inherently contains many obstacles created by partitions. In our case, a modular system has been used to create a building kit which consists of partitions of a fixed size, floor blocks and posts. The kit allows creation of different environments of rectangular layout. The color of the partitions, as well as of the floor, is white. The only exception is the upper edge of the partitions which can be of black or red color. The color of the posts is silver. The colors are relevant for analysis of the maze layout using the camera system [13].

Considering the standardized dimensions of the partitions and the rectangular layout of the maze, a grid of square cells of size $184 \times 184 \mathrm{~mm}$ can be formed over the maze. The size of the cells is determined by dimensions of the partitions and the posts. Here and farther, dimensions of any object are written as length $\times$ width $\times$ height. An example of a typical layout of the maze is shown in Fig. 1.

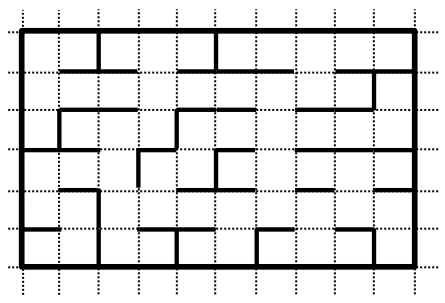

Fig. 1. Example of a possible layout of the maze. Partitions are symbolized using bold solid lines, the grid forming cells are depicted using thin dashed lines.

\section{B. Camera system}

The camera system consists of a stand arm and a camera. The camera is fixed on the stand arm and the whole camera system is placed such that the camera is above the maze. The camera can be used either for analysis of the maze layout or for localization of the robot [14]. However, information about position of the robot is used solely for the path-planning. No direct control of robot motion is assumed on the basis of this information. Control of the robot movement is performed by a microcontroller integrated in the robot. The scheme of the camera system installation is shown in Fig. 2.
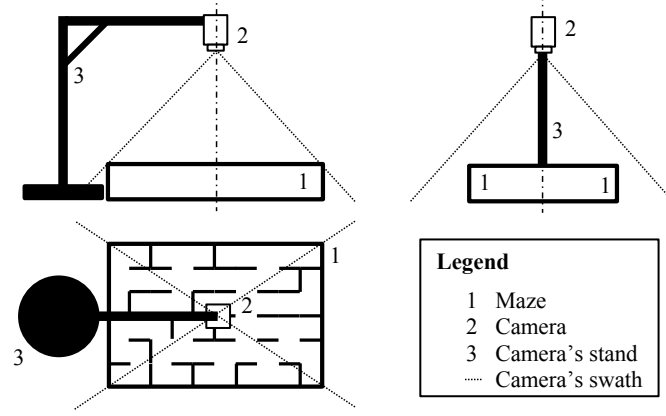

Fig. 2. Placement of the camera system with respect to the maze.

\section{Mobile robot}

Construction of the mobile robot has been restricted by the low-price requirement. Thus a differential wheeled robot of a simple construction has been developed. The robot is driven by two quadruple high-current half-H drivers L293D [15]. The speed of wheel rotation is measured using an incremental optical encoder attached on the inner side of the wheel where the reading is realized using reflective object sensors QRD1114 [16].

Detection of obstacles is realized on the basis of data from two front and two side reflectance sensors. They are based on infrared emitters L-53F3C and phototransistors L-53P3BT. The robot is also equipped with one triple axis magnetometer sensor HMC5883L [17] for detection of magnetic landmarks. Approximate placements of the sensors and actuators in the robot body are shown in Fig. 3 .

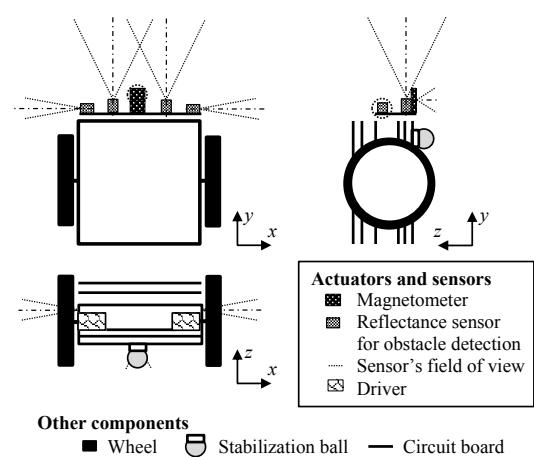

Fig. 3. Placement of the actuators and the sensors in the robot body. The reflective object sensors used for measurement of wheel rotation speeds and the Bluetooth module are not shown in this scheme. The coordinate system relative to the robot body is stated at each view.

Control of the entire robot, including data processing and communication, is ensured by microcontroller ATmega328P [18]. Specifically, Arduino UNO rev. 2 [19] has been used. Communication with other electronic devices is possible either via USB cable or via Bluetooth module HC-05 [20]. As is obvious in Fig. 4, the shape of the robot body is approximately a block of dimensions $110 \times 94 \times 83 \mathrm{~mm}$ (without the Bluetooth module)

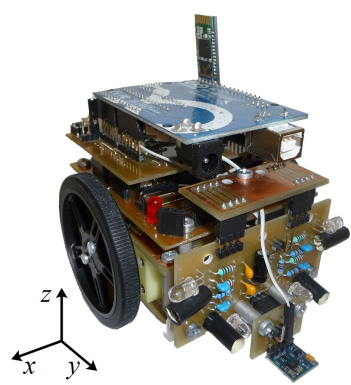

Fig. 4. The front of the robot; the coordinate system of the robot is shown bottom left.

\section{Path-planning and movement execution}

The robot is partially controlled by a high level control system, e.g. a computer, or a tablet. The high 
level control system processes image data from the camera system. On the basis of the data and users requirements, path-planning is performed. Different path-planning algorithms can be used either classical [21] or more specialized [22]. The most natural output of a path-planning algorithm is a plan described as a sequence of cells through which the robot should pass.

However, in our approach, the plan is required to be expressed as a sequence of instructions the robot should execute in the cells. For each cell, one instruction from a predefined set of instructions is assigned, where the set consists of the following instructions: 'keep direction', 'turn-left', 'turn-right', 'rotated through $180^{\circ}$ ', and 'stop'.

The robot implements the plan in an order given by the sequence. In each cell, one instruction is executed. Once an instruction is realized, a forward linear displacement of the robot to the center of the next adjacent cell is performed. In the center of this cell, the next instruction of the plan is executed and the whole process is repeated. Naturally, the instruction 'stop' breaks this loop.

\section{E. Concept of the localization}

The motion of the robot in the maze is required to be realized according to the loop which has been described in subsection II-D. The robot is equipped with a set of sensors. In our conception, all the sensors are used for the localization. The front and side reflectance sensors, as well as the encoders, are essential for execution of the forward linear displacement between two adjacent cells. However, information about cell borders must be provided by the magnetometer sensor.

The magnetic landmarks are used for labeling of borders in this conception. Considering dimensions of the robot and dimensions of the cells, landmarks of size $60 \times 4 \mathrm{~mm}$ are used in the presented solution. They are placed in the middle of each border such that the longer side of the landmark is parallel with the border (see Fig. 5). Such placement ensures that the robot will not miss any landmark.

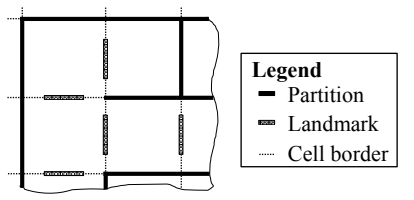

Fig. 5. Placement of the magnetic landmarks in the maze. The landmarks are placed on all borders of each cell in the middle of the border lengths; however, only landmarks not covered by partitions are visible in the scheme.

The landmarks are affixed on the floor according to the given conditions. They are made from a white magnetic sheet of thickness $1 \mathrm{~mm}$. The white color makes the landmarks invisible to the camera system. The width of the landmarks is the smallest possible, which still enables an easy installation, while keeping desired magnetic properties.

\section{IMPLEMENTATION OF THE MAGNETIC LOCALIZATION SYSTEM}

Although the introduced conception is simple, its practical implementation is not smooth. Even preliminarily static experiments (robot did not move during measurements) did not imply any problem; the first dynamic experiments (robot executed a movement during measurements) did not do well. Detection of the cell borders was not guaranteed by all of tested landmarks. However, our subsequent research helped us to find the essence of the issue, and subsequently to develop a robust solution.

A hypothesis explaining the nature of the issue is given in subsection III-A. Up to now, no detailed information about implementation of the magnetometer sensor was given. This lack is rectified in subsection III-B. Verification of the hypothesis is based on a practical experiment. It is described in subsection III-C. The experiment results are analyzed in subsection III-D.

A. Hypothesis about influence of landmark construction on overall performance of the localization system

In our approach, magnetic strips made from the commercial magnetic sheet are used as landmarks. The strips are hand manufactured as cuttings of the sheet. Magnetic sheets available on the market are axially magnetized. Thus the direction of magnetization changes every few millimeters, so the north and south poles are laid out in stripes as is shown in Fig. 6.

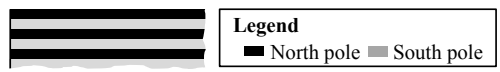

Fig. 6. Cutout of a magnetic sheet with indicated layout of the poles.

Let us suppose that the layout of the magnetic poles in a strip significantly influence performance of the localization system. Then a landmark with poles oriented along its length should have different properties than a landmark with poles perpendicular to the length. Let us label these two variants of landmarks as type A and type $B$, respectively. Orientations of the poles in the strips are shown in Fig. 7 for both types.

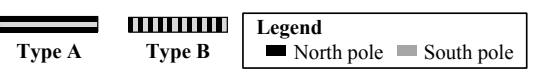

Fig. 7. Layout of the magnetic poles in the landmarks of type A and $\mathrm{B}$.

\section{B. Implementation of the magnetometer}

Since magnetic fields of commercial magnetic sheets are weak, a magnetometer sensor designed for lowfield magnetic sensing had to be used. In our case, a three axes magnetometer sensor HMC5883L [17] has been chosen. It has been placed out of the robot body, in the middle of the robot face, $5 \mathrm{~mm}$ above ground (floor of the maze). Such placement minimizes possible interferences caused by the robot. The sensor is parallel with the ground in the sense of its length and width.

With the microcontroller board [19], the sensor is connected via an $\mathrm{I}^{2} \mathrm{C}$ bus on ports $\mathrm{A} 4$ (SDA) and 
A5 (SCL). The used connection allows the reading of data for all three axes. Orientation of the coordinate system of the sensor coincides with orientation of the coordinate system of the robot (see Fig. 3 and Fig. 4), i.e. the $x$ and $y$ axes are parallel with the ground while $z$ axis is perpendicular to it.

\section{Experimental verification of the hypothesis}

It is assumed in the hypothesis that the layout of the poles in a magnetic strip significantly influences performance of the localization system. Verification of the hypothesis is based on a simple practical experiment. In this experiment, ability of the sensor to detect the examined types of landmarks, while the robot is in motion, is verified. Conditions of the experiment are described in further details.

At first, an examined landmark is fixed on the floor and the robot is placed in a predefined distance before the landmark in the sense of the movement direction as is shown in Fig. 8. The distance is given by the front edge of the sensor and middle of the landmark. Since, for some magnetic strips, the sensor is able to detect the magnetic field up to $30 \mathrm{~mm}$ in static, this value has been used as the distance in the experiment.

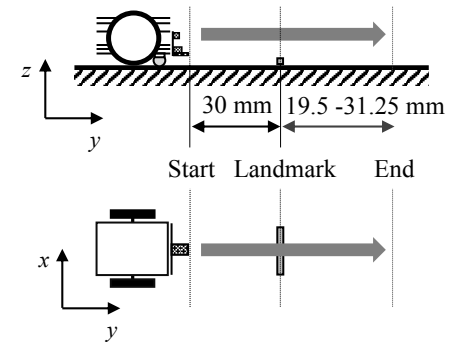

Fig. 8. Schematic representation of the experiment from the side view (upper part) and the top view (bottom part). At the beginning, robot is placed at the start position (the left side). During the experiment, the robot moves from the start position to the end position (the right side). An ideal trajectory of the movement is a line segment which passes through the geometric center of the landmark and is perpendicular to its length. The landmark is placed approximately in the middle of the trajectory.

During the experiment, the robot moves with a constant speed from the start position straight to end position. The end position is not determined by a distance from the landmark or the start; however, it is given by duration of the motion. The duration of the motion should be chosen so that the landmark is approximately in the middle of the trajectory. The trajectory is a line segment and it should pass through the geometric center of the landmark. Moreover, it should be perpendicular to the longer side of the landmark. These requirements match with expected behavior of the robot in the maze (see subsection II-D).

The capability of the sensor to detect the landmarks is examined at three different speeds: $v_{1}=$ $0.060 \mathrm{~m} \mathrm{~s}^{-1}, v_{2}=0.098 \mathrm{~m} \mathrm{~s}^{-1}$, and $v_{3}=0.120 \mathrm{~m} \mathrm{~s}^{-1}$, where $0.120 \mathrm{~m} \mathrm{~s}^{-1}$ is the maximum speed which guarantees maintaining of the movement direction. Higher speed may cause oscillations of the robot or jumping aside while passing a landmark. For all the speeds, the sample time $25 \mathrm{~ms}$ has been used for reading of the data from the sensor.

The experiment has been realized ten times for each landmark and each speed. In total, six landmarks have been created, three of each type. As was mentioned in subsection III-A, the landmarks are strips made from the magnetic sheet. Since magnetic properties of the sheets may vary over its surface, three pairs of landmarks have been made, each pair from different part of the sheet. For clarification, one pair consists of one landmark of the type A and one of the type B.

\section{Evaluation of the experiment results}

In total, one hundred eighty measurements have been realized within the experiment where the measured data is a time series of magnetic flux $B$. Under ideal conditions, in the time $t$, the presence of a landmark nearby the sensor is recognized as a significant change of the magnetic flux $B(t)$, in compare to a normal state.

Naturally, the magnetic flux of a landmark does not appear equally in all directions. However, the used sensor is able to sense the flux in all three dimensions. Using of all available data may increase probability of the landmark detection. This potential has been considered in the experiment, and accordingly measurements of the magnetic fluxes along the $x, y$ and $z$ axes, $B_{x}(t), B_{y}(t)$ and $B_{z}(t)$, were performed.

A preliminary analysis of the experimental data has shown that no significant variation of $B_{x}(t)$ has been recorded within the experiment. It is consistent with the conditions of the experiment (trajectory is a line segment perpendicular to the landmark). Since this data does not contain any usable information, it is excluded from the next analysis.

However, the preliminary analysis has confirmed expected responses on proximity of a landmark in the sense of the $z$ axis. This is the same case for both types of landmarks. Moreover, for the type A, responses along the $y$ axis were recorded, too. For illustration, examples of detected responses for the type A and the type B are shown in Fig. 9 and Fig. 10, respectively.

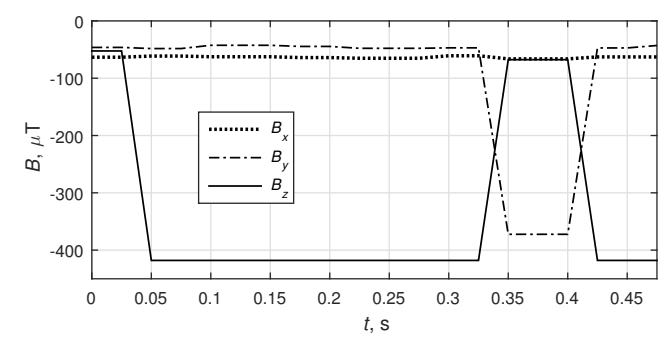

Fig. 9. Magnetic fluxes recorded by the sixth measurement for the landmark of the type A which belongs to the second pair of landmarks. The motion speed was $0.120 \mathrm{~m} \mathrm{~s}^{-1}$.

As is obvious from the graphs shown in Fig. 9 and Fig. 10, proximity of a landmark is indicated as a change of the magnetic flux from a value around $-55 \mu \mathrm{T}$ to the value $-418 \mu \mathrm{T}$ in the direction of the $z$ axis. For the type A, change of the flux from a value around $-45 \mu \mathrm{T}$ to the value $-372.4 \mu \mathrm{T}$ occurs along 


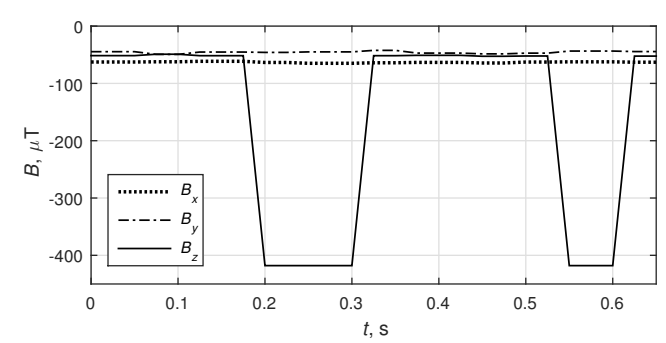

Fig. 10. Magnetic fluxes recorded by the sixth measurement for the landmark of the type B which belongs to the second pair of landmarks. The motion speed was $0.098 \mathrm{~m} \mathrm{~s}^{-1}$.

the $y$ axis, too. Supposing these facts, let us express proximity of a landmark as a binary variable

$$
l(t)= \begin{cases}1 & \text { if } B(t)<-300 \\ 0 & \text { otherwise }\end{cases}
$$

where $l(t)=1$ occurs when proximity of a landmark is detected in the time $t$, otherwise $l(t)=0$. This equation holds for both considered magnetic fluxes, i.e. for $B_{y}(t)$ and $B_{z}(t)$. Consequently, the proximities are marked as $l_{y}(t)$ and $l_{z}(t)$, respectively.

Within the experiment, the basic requirement on the localization system is to detect presence of a landmark on the trajectory. For that purpose, the proximities $l_{y}(t)$ and $l_{z}(t)$ can be used. Let us consider that the presence of a landmark is confirmed by at least one non-zero value in the time series $l(t)$. Then the presence of a landmark $p$ is a binary variable given by

$$
p=\max _{k \in T} l(k),
$$

where $T$ is a set of all time instances $k$ when the measurement has been performed. The set $T$ is given by the used sample time and duration of movement. In the case a landmark is detected on the basis of the measured data, $p=1$ otherwise $p=0$. In accordance with the used marking, the presence of a landmark detected on the base of $l_{y}(t)$ is donated as $p_{y}$. The same logic is used for $p_{z}$.

A simple performance measure can be defined on the basis of the variable $p$. The measure is given as number of measurements in which presence of the landmark was detected. In our case, two measures directly arise: the number of detections in direction of the $y$ axis $\sum_{i=1}^{n} p_{y}^{(i)}$ and the number of detections in direction of the $z$ axis $\sum_{i=1}^{n} p_{z}^{(i)}$ where the upper index $(i)$ denotes the $i$-the measurement performed for a tested landmark by one of the considered speeds, and $n=10$ for all the speeds and all tested landmarks.

As was already mentioned, probability of the detection can be increased by combining data measured in different directions. Thus let us suppose a landmark to be detected once its presence is confirmed either in the $y$ or the $z$ axis direction. On the basis of this assumption, a third measure can be defined as $\sum_{i=1}^{n}\left(p_{y}^{(i)} \vee p_{z}^{(i)}\right)$. All the introduced measures have been used by evaluation of the experimental data. The evaluation results are summarized in the following three tables.
The detection performance in the sense of the $y$ axis is summarized in Table I. In the first column, indices of samples (label pairs) are stated. In the remaining columns, achieved scores are given for both types of landmarks and all the speeds. The same structure is used also in the other tables.

TABLE I

SCORE OF THE LOCALIZATION SYSTEM WHILE $B_{y}(t)$ IS USED BY THE LANDMARKS DETECTION, EVALUATED USING THE MEASURE $\sum_{i=1}^{10} p_{y}^{(i)}$

\begin{tabular}{ccccccc}
\hline Sample & \multicolumn{3}{c}{ Type A } & \multicolumn{3}{c}{ Type B } \\
No. & $v_{1}$ & $v_{2}$ & $v_{3}$ & $v_{1}$ & $v_{2}$ & $v_{3}$ \\
\hline 1 & 10 & 10 & 10 & 0 & 0 & 0 \\
2 & 10 & 10 & 9 & 0 & 0 & 0 \\
3 & 10 & 10 & 10 & 0 & 0 & 0 \\
\hline
\end{tabular}

It is obvious from the data in Table I that only the presence of a landmark of the type A can be detected on the basis of $B_{y}(t)$. Nevertheless, the presence of a landmark of the type A was detected almost without any mistake. Only for the sample No. 2, the proximity of the landmark was correctly recognized nine times at the speed $v_{3}$. Just as reminder, the maximum score is ten for all the speeds and all the tested landmarks.

Table II contains scores achieved, once the data measured in the $z$ axis direction is used solely. For the samples No. 2 and No. 3, detection of landmarks of both types was successful for all the measured data. However, poor performance is noticeable for the landmarks in sample No. 1. The performance is worsening with increasing speed of motion.

TABLE II

SCORE OF THE LOCALIZATION SYSTEM WHILE $B_{z}(t)$ IS USED BY THE LANDMARKS DETECTION, EVALUATED USING THE MEASURE $\sum_{i=1}^{10} p_{z}^{(i)}$

\begin{tabular}{ccccccc}
\hline Sample & \multicolumn{3}{c}{ Type A } & \multicolumn{3}{c}{ Type B } \\
No. & $v_{1}$ & $v_{2}$ & $v_{3}$ & $v_{1}$ & $v_{2}$ & $v_{3}$ \\
\hline 1 & 9 & 9 & 6 & 10 & 9 & 7 \\
2 & 10 & 10 & 10 & 10 & 10 & 10 \\
3 & 10 & 10 & 10 & 10 & 10 & 10 \\
\hline
\end{tabular}

The combination of the data measured in the direction of the axes $y$ and $z$ improves performance of the system by detection of landmarks of the type A. It is clearly visible while comparing Table III with Table I and Table II. For the type A, the landmarks were correctly recognized in all the datasets. However, no improvement can be observed for landmarks of the type B. Indeed, there is no relevant information in $B_{y}(t)$ for this type of landmark.

The results summarized in Table I and Table II also indicate another interesting fact. As was expected, the quality of the magnetic sheet is not equal over the whole surface. This is clearly visible while comparing achieved scores of samples No. 2 and No. 3 with the scores of sample No. 1. Although magnetic properties of the landmarks belonging to the sample No. 1 are not ideal, the combination of the data measured in the 
TABLE III

SCORE OF THE LOCALIZATION SYSTEM WHILE $B_{y}(t)$ AND $B_{z}(t)$ ARE USED SIMULTANEOUSLY BY THE LANDMARKS DETECTION, EVALUATED USING THE MEASURE $\sum_{i=1}^{10}\left(p_{y}^{(i)} \vee p_{z}^{(i)}\right)$

\begin{tabular}{ccccccc}
\hline Sample & \multicolumn{3}{c}{ Type A } & \multicolumn{3}{c}{ Type B } \\
No. & $v_{1}$ & $v_{2}$ & $v_{3}$ & $v_{1}$ & $v_{2}$ & $v_{3}$ \\
\hline 1 & 10 & 10 & 10 & 10 & 9 & 7 \\
2 & 10 & 10 & 10 & 10 & 10 & 10 \\
3 & 10 & 10 & 10 & 10 & 10 & 10 \\
\hline
\end{tabular}

direction of the $y$ and the $z$ axes, guarantees excellent performance for landmarks of the type A.

Considering the stated experimental results, the most robust solution, based on the introduced idea, should be to solely use magnetic landmarks of the type A, and their detection should simultaneously use both magnetic fluxes, $B_{y}(t)$ and $B_{z}(t)$.

\section{CONCLUSION}

In this paper, a low-cost localization system designed for indoor mobile robots has been introduced. The system has been designed for a teaching aid where a mobile robot operates in a maze. The introduced localization system is one of the essential parts which allow semi-autonomous behavior of the robot.

The localization system is based on magnetic landmarks which are detected by a low-cost triple axis magnetometer sensor. The landmarks are made of commercial magnetic sheets. Such created landmarks are cheap, easily available and simply applicable. In combination with the used sensor, they create a reliable localization system under some conditions. Besides a proper placement of the sensor, placement of the landmarks, or appropriate setting of the sample time, there is one trickier requirement.

The unexpected requirement arises from construction of the magnetic sheets. The magnetic sheets available on the market are axially magnetized. Since the landmarks are manually cut out of the sheet, the layout of the magnetic poles in the landmarks is influenced by the manufacturing process. The experiment presented in this paper has shown that performance of the localization system is influenced by the layout of the poles in the landmarks.

On the basis of the experiment results, the following conclusion can be made. The suggested localization system based on the magnetic strips can be reliable; however, the layout of the poles in the landmarks should be parallel with their longer side. Supposing the trajectory of the robot motion, which is the line segment perpendicular to the landmark, a change of magnetic fluxes in the direction of the $y$ and the $z$ axis can be detected by the sensor near such landmarks. To increase robustness of the system, simultaneous using of these two signals by the landmarks detection is recommended. Following these insights, implementation of the introduced localization system into the control algorithm of the mobile robot will be accomplished in the near future.

\section{ACKNOWLEDGMENT}

The work has been supported by the Funds of University of Pardubice, grant No. SGS-2016-017.

\section{REFERENCES}

[1] M. Pakdaman and M. M. Sanaatiyan, "Design and implementation of line follower robot," in Computer and Electrical Engineering, 2009. ICCEE '09. Second International Conference on, vol. 2, Dec 2009, pp. 585-590.

[2] S. F. R. Alves, H. Ferasoli Filho, R. Pegoraro, M. A. C. Caldeira, J. M. Rosário, and W. M. Yonezawa, Research and Education in Robotics - EUROBOT 2011. Springer Berlin Heidelberg, 2011, ch. Educational Environment for Robotic Applications in Engineering, pp. 17-28.

[3] M. P. Cuéllar and M. C. Pegalajar, "Design and implementation of intelligent systems with LEGO mindstorms for undergraduate computer engineers," Computer Applications in Engineering Education, vol. 22, no. 1, pp. 153-166, 2014.

[4] R. M. Harlan, D. B. Levine, and S. McClarigan, "The khepera robot and the krobot class: A platform for introducing robotics in the undergraduate curriculum," SIGCSE Bull., vol. 33, no. 1, pp. 105-109, Feb. 2001.

[5] M. Rubenstein, C. Ahler, N. Hoff, A. Cabrera, and R. Nagpa, "Kilobot: A low cost robot with scalable operations designed for collective behaviors," Robotics and Autonomous Systems, vol. 62, no. 7, pp. 966-975, 2014.

[6] M. Mariška and P. Doležel, "Multi agent environment for modelling and testing of cooperative behaviour of agents," Advances in Intelligent Systems and Computing, vol. 289, pp. 301-306, 2014

[7] A. Chatterjee, A. Rakshit, and N. N. Singh, Vision Based Autonomous Robot Navigation: Algorithms and Implementations. Springer Berlin Heidelberg, 2013, ch. Mobile Robot Navigation, pp. 1-20.

[8] J. Huh, K. Lee, W. K. Chung, W. S. Jeong, and K. K. Kim, "Mobile robot exploration in indoor environment using topological structure with invisible barcode," in Intelligent Robots and Systems, 2006 IEEE/RSJ International Conference on, Oct 2006, pp. 5265-5272.

[9] H. Chae and S. Na, "Mobile robot navigation using rfid and vision sensor," in Information Technology Convergence, 2007. ISITC 2007. International Symposium on, 2007, pp. 52-57.

[10] A. Lazanas and J. C. Latombe, "Landmark-based robot navigation," Algorithmica, vol. 13, no. 5, pp. 472-501.

[11] B. J. Choi, B. Kim, S. M. Jin, J. C. Koo, W. K. Chung, H. R. Choi, and H. Moon, "Magnetic landmark-based position correction technique for mobile robots with hall sensors," Intelligent Service Robotics, vol. 3, no. 2, pp. 99-113, 2010.

[12] W. S. You, B. J. Choi, B. Kim, H. Moon, J. C. Koo, W. Chung, and H. R. Choi, "Global localization for a small mobile robot using magnetic patterns," in Robotics and Automation (ICRA), 2010 IEEE International Conference on, 2010, pp. 2618-2623.

[13] P. Škrabánek, "Labyrinth arrangement analysis based on image processing," in Mendel 2015, ser. Advances in Intelligent Systems and Computing, vol. 378. Springer International Publishing, 2015, pp. 305-316.

[14] P. Škrabánek and P. Doležel, "Attractive robot's design suitable for image processing," in Proceedings of the Mendel 2014: 20th International Conference on Soft Computing. University of Technology Brno, 2014, pp. 217-222.

[15] Quadruple high-current half-H driver L293D, Texas Instruments Inc., January 2016.

[16] QRD1113 / QRD1114 - Reflective Object Sensor, Fairchild Semiconductor Corporation, June 2013, rev. 1.2.0.

[17] 3-Axis Digital Compass IC HMC5883L, Honeywell International Inc., February 2010, rev. E.

[18] 8-bit AVR Microcontroller with 8/16/32K Bytes in-system programmable flash, Atmel Corporation, 2010, rev. CORP072610.

[19] "Arduino UNO \& Genuino UNO," mar 2016. [Online]. Available: https://www.arduino.cc/en/Main/ArduinoBoardUno

[20] Module Data Sheet HC-05, Guangzhou HC Information Technology Co., Ltd., April 2011, rev. 1.01.

[21] S. Russell and P. Norvig, Artificial Intelligence: A Modern Approach, 3rd ed. Prentice Hall Press, 2009.

[22] P. Škrabánek, M. Mariška, and P. Doležel, “The time optimal path-planning of mobile robots motion respecting the time cost of rotation," in Proceedings of the 20th International Conference on Process Control. STU Bratislava, 2015, pp. 232-237. 